\title{
TAGUNGSBEITRÄ GE
}

WIENER SLAVISTISCHES JAHRBUCH, Band 53/2007, 9-25

(C) 2007 by Österreichische Akademie der Wissenschaften, Wien

HEINZ DIETER POHL

\section{Die Bedeutung des Slowenischen für die Toponymie der Tauernregion}

\begin{abstract}
Slovanska jezikovna oblika kot podlaga krajevnih imen na vzhodu in jugu Avstrije (Vzhodna Tirolska, Koroška, avstrijska Štajerska, Salzburg-Lungau, južni del Spodnje Avstrije ter vzhodni del Gornje Avstrije) ustreza ,alpski slovanščini““ oz. jeziku staroslovenskih „Brižinskih spomenikov“. Nemške variante slovanskih/slovenskih imen je v glavnem mogoče razložiti kronološko, iz česar sledi, da je v Avstriji v visokem in poznem srednjem veku preko okvirov Koroške vladala močno razširjena slovansko-nemška jezikovna mešanica, kar se odraža tudi v starih izposojenkah. To jezikovno mešanico slovanstva v Avstriji zgodnjega srednjega veka pokažejo jasno imena v „Narodnem Parku Visokih Tur“ ter v občinah Kals am Grossglockner in Heiligenblut.
\end{abstract}

\section{Allgemeines; 2. Kals am Grobglockner; 3. Das Gössnitztal; 4. Einige interessante Bergnamen aus dem Nationalpark Hohe Tauern}

1.0. Die meisten Österreicher verbinden mit dem Wort „slowenisch“ meist die Republik Slowenien, in zweiter Linie auch Kärnten - insbesondere mit dem Minderheitenproblem und Ortstafelkonflikt. Weniger bekannt ist die Tatsache, dass die Slowenen ein Teil unserer österreichischen Geschichte sind und viele Spuren in jenen Gegenden hinterlassen haben, in denen die slowenische Sprache schon lange verklungen ist, wo man aber im Namengut und in vielen (teils mundartlichen) Lehnwörtern immer wieder unserem sprachlichen slowenischen Erbe begegnet.

Die Symbiose zwischen den zunächst in die alpinen Gebiete Österreichs eingewanderten (,karantanischen“) Slawen und den später zugewanderten Baiern war sehr eng. Die Eingliederung des (slawischen) karantanischen Fürstentums in das bairische Herrschaftsgebiet wirkte sich auch sprachlich aus. Einerseits setzten sich die bairisch-österreichischen Dialekte als lokale Verkehrssprachen immer mehr durch, andererseits hinterließ die slawische Grundschicht zahlreiche Wörter in der deut- 
schen Mundart. Die meisten spezifisch „karantanischen“ Wörter sind Bergappellativa oder Begriffe aus dem Bereich der bäuerlichen Sphäre (Pflanzen und Speisen). ${ }^{1}$

Typische Bergappellativa sind u. a. bordo 'Berg, Eck' (BN Pyhrn, Birnig, SN Verditz), duplo 'Höhle' (BN Tauplitz), dъbrb 'Schlucht, Klamm' (in Daber-, BN Dobratsch), gazъ/-b 'Über-, Durchgang; ausgetretener Weg' (BN Pyhrgas sub 1.4, SN Wigasnitz / Vijasce Vigasce), gorica 'Bühel, Bichl' (BN Gerzkopf, Göritz), gričb 'Hügel, Steile, Anhöhe' (BN Gritsch), lanežb '*Gletscher' (BN Lanischeck, -kees, Wainasch / Vajnež), prědělı 'Wasserscheide; Grenze' (SN Predl, GN Brettlbach), sotb 'Gebirgsweg' (BN Sattnitz / Sotnica Gure), stodorb 'Felsgrund' (BN Gstoder, Stadurz). - Aus der bäuerlichen Sphäre sollen hier u. a. erwähnt werden: chyša 'Hütte, Haus' (> dt. Keusche, älter Kaixen), čblnъ 'Kahn' (> dt. Zille), južina 'Mittagessen' ( $>$ dt. Jause 'Zwischenmahlzeit', im Lesachtal alt 'Mittagessen'), kyselica 'Gesäuertes' ( $>$ dt. Gaislitz 'Speise aus Hafermehl'), moka 'Mehl' ( $>$ dt. Munggen 'eine einfache Speise aus Hafer- und Gerstenmehl'), oblica 'Rübe' ( $>$ dt. Oblitzen), pograd 'Gerüst an der Wand, Pritsche' (> dt. Pograte(n) 'einfache Schlafstelle, Bretterboden'), *p(r)ętro bzw. *p(r)ętra 'Art Gestell aus Brettern', slow. peter / petro 'Heuboden' ( $>$ dt. Pränter, Gepater 'Oberboden der Scheune'), *strak-, slow. strok(a) (> dt. Strankerl 'grüne Bohne, Fisole'), *tălkŭnă 'Hafermehl' (> dt. Talggen 'Hafergrütze, -brei'), *topenica 'Erwärmtes, Erhitztes' ( $>$ dt. Topanitz 'eine Art aufgeblähte Brotschnitte, Toast', Kals).

In der verklungenen alpenslawischen Sprache gab es auch deutsches Wortgut beide Sprachen bereicherten einander also gegenseitig - wie dies eben in allen gemischtsprachigen Regionen zu beobachten ist. Als Beispiel sei hier der Wortschatz angeführt, der die bestehenden Herrschaftsstrukturen beschreibt und der den Kern der Untersuchung Holzers ${ }^{2}$ zur Sprache der Slawen in Österreich im Mittelalter darstellt. Die von ihm behandelten slawischen Wörter reflektieren zum Großteil speziell das Grundherrschaftswesen im bairischen Ostland. Die Wörter gehören verschiedenen Kategorien an, Entlehnungen aus dem Deutschen sind losb (Terminus der Landzuteilung, zu dt. Los) ${ }^{3}$ und mytaŕs 'Mauteinnehmer' (zu mhd. mütaere), Lehnübersetzungen sind službba 'Dienst (servitium)', strělbcb 'Schütze', militärischer Terminus, geschrieben Strelz o. ä. und (vielleicht) poklons 'Abgabe, Geschenk (eigentlich Verneigung ${ }^{4}$ )', geschrieben poklon, poglon, auch pogklann; ein slawisches Lehnwort im Bairischen ist županъ 'Vorsteher, Amtmann, Dorfrichter' (eigentlich 'Ältester'), eingedeutscht Suppan (erhalten als Familienname), eventuell auch einige andere. ${ }^{6}$

${ }^{1}$ Auch Lehnübersetzungen wie Füchsling 'Eierschwammerl, Pfifferling' (wie slow. lisička zu lisica 'Fuchs', sonst sagt man in den bairisch-österreichischen Mundarten neben Eierschwammerl eher Rehling oder Reherl) - vgl. Pohl 2004: 67 u. 202.

2 Holzer 2002a u. 2002c (alle Begriffe werden näher erläutert; hier sind nicht alle angeführt).

3 FN Losbnica (vgl. Holzer 2001: 75 f.).

4 Analog dt. Verehrung nach lat. honorarium (Holzer 2002: 64).

5 Näheres s. Holzer 2001: $98 \mathrm{f}$.

${ }^{6}$ Vgl. Holzer 2002: 68. 
Aus dem karantanischen Bereich wäre auch noch *kosez 'Edling (bevorrechteter Bauer, Freisasse)' sowie ban 'ein Fürstentitel' zu erwähnen. ${ }^{7}$

Was das slawische Lehngut Österreichs insbesondere im Bereich der Hohen Tauern betrifft, ist schon eingangs festzuhalten, dass es sich auf Grund linguistischer Fakten als größtenteils sehr alt erweist, obwohl das Meiste davon erst relativ spät überliefert wird. Dies zeigen deutlich einige lautliche Eigenschaften, wie z. B. einige Fälle von frühslaw. $a$ (genauer $\breve{a}$ ) für späteres $o$, Fehlen des prothetischen $j$-, eindeutige Spuren der Nasalvokale, teilweises Unterbleiben der Liquidametathese sowie der Reflex -schk- für slow. -šč-.

1.1. Fälle von - $a$ - liegen vor u. a. in (860) Astaruuiza 'Hochosterwitz' (zu slaw. oster) und 1005 Adamunta 'Admont' (zu slaw. *odbmot-), vielleicht in Stadurz (zu slaw. stodorb), weiters sub 3 Kasaze u. Kasarn sowie Malesischk sowie sub 4 Gamolnigspitz(e); auch in einigen frühslawischen Lehnwörtern, wie u. a. Arl 'Hakenpflug' (aus slaw. *ărdlă), Kraxe 'Rückentrage' (aus slaw. *krăšńa) und Talggen 'Hafermehl' (aus slaw. *tălkŭnă).

1.2. Sprachgeographisch ist das prothetische $j$ - vom Zentrum des slawischen Sprachgebietes ausgegangen und an den Rändern entweder nicht konsequent oder überhaupt nicht durchgeführt worden. Solche Randlagen sind u. a. Oberkärnten und Osttirol (letzteres durchwegs ohne j-), z. B. Arnig (Gem. Kals) und Auernig (BN, Gem. Mallnitz), beide < slaw. *avorbnikb zu slow. javor 'Ahorn' gegenüber gleichbedeutenden Jauernigg (HN, südlich Predlitz, St) oder Jauring (SN/GN, Gem. Aflenz). Das benachbarte Aflenz (<*ablan(bn)ica, 1025 Auelniz) weist kein $j$ - auf, was eindeutig in der Chronologie begründet ist. ${ }^{8}$ Weiters in Osttirol und Oberkärnten Amlach (zu slaw. jama), in Osttirol Aßling (zu slaw. *asenb 'Esche').

1.3. Erhalt der Nasalvokale: Der Nasal $e$ ist auffallend selten belegbar (z. B. BN Lending, Granatspitzgruppe, Osttirol < *lędina oder -nikb 'Brachland', SN Fentsch, Gem. St. Marein bei Knittelfeld, St $<* V e t j-$, zu *Vętjeslavb wie u. a. altčech. Váceslav 'Wenzel', russ. Vjačeslav usw. 'größeren Ruhm habend'), $Q$ ist sehr häufig und weist manchmal noch die ältere $a$-Färbung auf, z. B. slaw. *loka 'feuchte Wiese' in Landschütz (FN, Gem. Matrei i. O.), Lonza (BN, Gem. Mallnitz), Lang (2x, SN, Gem. Ferndorf und Feldkirchen), Wangenitzen bzw. Wangenitztal oder Sunzkopf (sub 4), Langen (SN, Gem. St. Andrä), Lansach (SN, Gem. Weißenstein), Laing (SN, Gem. Spielberg bei Knittelfeld), Lang (SN, Gem., Leibnitz). In jüngeren slow. (also relativ spät ins Deutsche gelangten) Ortsnamen ist kein Nasalvokal mehr vorhanden, z. B. Laak / Loka (SN, Gem. Ferlach). Der Erhalt der Nasalvokale widerspiegelt das Alter des Namengutes. Je früher ein Name ins Deutsche gelangt ist, des-

7 Zur Etymologie u. a. Bezlaj 1976: 11 u. 1982: 69. Beide wohl türk.-awarischer Herkunft (so auch Kranzmayer 1956: 62 ff.), *kosez verwandt mit russ. kozak sowie türk. Kazach.

8 Wie u. a. auch dt. Aßling gegenüber Jesenice (zu *asenb bzw. slow. jesen 'Esche') in Slowenien. Vgl. auch die Karte 2 bei Mader 1986. 
to eher erscheint ein Reflex des Nasalvokales, übrigens auch in PN, z. B. Zuentipolch 'Svętipъlkъ / Svatopluk usw.', Zanto / Zunducu 'Sodъ(kъ)' zu sods 'Richter' (wohl Kurzform zu einem zusammengesetzten PN, im SN Zandlach, Gem. Reißeck). ${ }^{9}$ Ein besonderes Charakteristikum der Slavia submersa ist darin aber nicht zu erblicken, ${ }^{10}$ doch es ist ein Zeugnis hoher Altertümlichkeit, die auch alte Lehnwörter wie Munggen (zu slaw. moka 'Mehl') oder Strankerl (zu slaw. *strak-, slow. Stro$k(a)$ ) sowie Pränter (zu slaw. *p(r)ętro bzw. *p(r)ętra 'Art Gestell aus Brettern') zeigen.

1.4. Unterblieben ist die Liquidametathese u. a. in Virgen / Firschnitz (zu slaw. *bergъ bzw. *beržbnica), HN Perloger (Gem. Kals im Gegensatz zu 1410 Prelug in Ainet, zu slow. prelog 'Brachland'), BN Pyhrgas (< slaw. *per-gaz- 'Durchgang'), sowie die sub 1.1. genannten alten Lehnwörter Arl und Talggen.

1.5. Die Lautung -schk- ist in Oberkärnten, Osttirol und im Salzburger Lungau die Regel, sie ist v. a. im Wortbildungselement (nomina loci) slow. -išče besonders häufig. Wenn man das Material betrachtet, entsteht der Eindruck, Oberkärnten und Osttirol sowie Salzburg (v. a. Lungau) und Teile der Steiermark hätten $s(c h) k$, aber Unterkärnten und der größere Teil der Steiermark $s t$ - Reflex alpenslawischer dialektologischer Verhältnisse. ${ }^{11}$ Doch vielmehr scheint es so gewesen zu sein, dass ursprünglich überall das altslow. šć sehr palatal ausgesprochen wurde und in früh eingedeutschten Gebieten als schk übernommen wurde wie z. B. Staniska (SN, Kals < *stanišce 'Standort, Lagerplatz') in Osttirol oder Patschgen (SN/FN, Gastein < *pašišce 'Weideplatz') in Salzburg. (insg. über 20 solche Belege). Dann ist diese Lautgruppe in Richtung neuslow. $\check{s} c$ hin entwickelt worden, was dann im Deutschen mit $\check{s} t$ wiedergegeben wurde bzw. im größten Teil des Bairischen st ergab, daher haben wir z. B. Gassarest (SN, Gem. Strassburg < *kozarišće 'Ort der Ziegenhirten') in Unterkärnten oder Liesing (GN/SN < *lěšćsnica zu lěsъkъ 'Haselnuss', 9./10. Jhdt. Liestinicha) in der Steiermark. ${ }^{12}$

1.6. In einem größeren Zusammenhang entspricht die slawische Sprachform, die den Toponymen im Osten und Süden Österreichs (Osttirol, Kärnten, Steiermark, Salzburg-Lungau, südliches Nieder- ${ }^{13}$ und östliches Oberösterreich) zu Grunde liegt, dem „Alpenslawischen“ (nach Ramovš ${ }^{14}$, Kronsteiner ${ }^{15}$ usw.) bzw. der Sprache der

\footnotetext{
9 Kronsteiner 1975: 65.

10 Zumal in den Jauntaler Mundarten bis heute die Nasalvokale z. T. erhalten sind (vgl. Zdovc 1972: $74 \mathrm{ff}$.)

11 Dies habe ich voreilig in Pohl 1996a: 312 f. behauptet, Ramovš (1936: 53) folgend.

12 In den meisten Kärntner slowenischen Mundarten ist $\check{s} \breve{c}>\check{s}$ geworden wie z. B. Homölisch / H(o)mel(i)še $(\mathrm{SN}$, Gem. St. Margareten im Rosental und Zell < *xъméljišćce zu slow. hmelj 'Hopfen'), eine Lautung, die auch der Steiermark nicht fremd ist, z. B. Fladi13 scherhof (HN, Gem Turnau, Bruck a. d. Mur < *blatišće zu slow. blato 'Sumpf').

13 Dazu vgl. Holzer 2001.

14 V. a. Ramovš 1936: 23 ff.

15 V. a. Kronsteiner 1975: $140 \mathrm{ff}$.
} 
altslowenischen „Freisinger Denkmäler“ ${ }^{16}$ was auch bereits der große slowenische Dialektologe und Sprachhistoriker Ramovš festgestellt hat. Die Varianten in der deutschen Wiedergabe der slawischen Namen wollte er dialektologisch deuten, doch eher sind diese Unterschiede chronologisch zu erklären, woraus folgt, dass es im hohen und späten Mittelalter eine über Kärnten hinausgehende weit verbreitete Gemischtsprachigkeit in Österreich gab ${ }^{17}$ was sich auch in alten Lehnwörtern widerspiegelt. ${ }^{18}$ Diese Sprache weist einige Besonderheiten auf, die sich in den slowenischen Kerngebieten nicht finden, u. a. den Ortsnamentyp auf -iče und das häufige Wortbildungselement -nik (eingedeutscht meist -nig) sowie einige Wörter. ${ }^{19}$ Es erhebt sich nur die Frage, wie man diese Sprache nennen soll, „slowenisch“ im heutigen Sinn ist sie nicht, ,alpenslawisch“ ist zu allgemein, daher denke ich, der beste Name wäre Karantanisch, da die Slavia submersa der österreichischen Alpenländer die Sprache des Karantanen-Reiches war, ${ }^{20}$ der ersten historischen Staatsgründung der Vorfahren der heutigen Slowenen.

2.0. Die Gemeinde Kals am Großglockner in Osttirol gehört zu den namenkundlich bemerkenswertesten Regionen Österreichs: hier haben im Mittelalter Romanen, Slawen und Deutsche friedlich nebeneinander gerodet, gewirtschaftet und gelebt (wie dies treffend Maria Hornung wiederholt festgestellt hat), ${ }^{21}$ was sich im Namenschatz des Kalser Tales noch heute deutlich zeigt, denn wir finden Namen romanischer (ladinischer) ${ }^{22}$, slawischer (alpenslawischer bzw. altslowenischer) ${ }^{23}$ und deutscher (südbairischer) Herkunft. Diese Vielfalt betrifft nicht nur die (amtlichen) Siedlungs- und Hofnamen, sondern auch die Flur-, Berg- und Gewässernamen.

2.1. Der Name Kals (urkundlich 1197 de Calce, 1240-48 Kals, 1256 Calts, 1329 Chalt(e)s, 1545 Kallss) ist wahrscheinlich auf (alt)slowenisch kalec $(<$ kalbcb, zu slawisch kalb 'Kot, Schlamm; Lache, Pfütze; Viehtränke', im Slowenischen kal 'Kot in Pfützen; flache Vertiefung, in der sich Regenwasser ansammelt, Lache; Viehtränke') bzw. kaluža '(Kot-) Lache, Pfütze' zurückzuführen. Man vergleiche

16 Dazu vgl. zuletzt Pohl 2002a: 177 ff. (mit Lit.) sowie 2002b: 53 ff. (mit Lit.).

17 Worauf in letzter Zeit auch Holzer in zahlreichen Arbeiten hingewiesen hat (s. Holzer 2002a-c).

18 Zum deutsch-slawischen Sprachkontakt in Österreich s. Pohl 1997 u. Neweklowsky 1997.

19 Dazu schon früher Pohl 2002a: v. a. 179 bzw. 2002b: 68 f.

20 Zur Begrenzung s. Pohl 2002a: 184 f. (mit Lit.) u. 2002b: 73, vgl. auch Holzer 2001: 50

21 Vgl. Hornung 1964: $78 \mathrm{f}$.

22 In den Alpen entstand eine eigene romanische Sprache, ,rätoromanisch“ oder „ladinisch“; sie ist in mehreren Varianten vom Schweizer Kanton Graubünden („Bündnerromanisch“) über Südtirol („Ladinisch“) bis nach Friaul („Furlanisch“) verbreitet und hat keine ein-

heitliche Schriftsprache entwickelt.

23 Die seit dem 7. Jhdt. in den Alpen nachweisbaren Slawen sind Verwandte (z. T. auch Vorfahren) der heutigen Slowenen; deren nur aus Namen bekannte und erschließbare Sprache bezeichnet man "Alpenslawisch“ oder „Altslowenisch“, sie steht der in den „Freisinger Denkmälern“ (ältestes slawisches Denkmal in lateinischer Schrift) verwendeten Sprache sehr nahe. 
damit heutige slowenische Siedlungsnamen wie Kalce, Kaľ̌e, Kališe und Kaluže. Der Name der Gemeinde kommt vom Namen des Kalser Tales, ursprünglich wohl ein Gegendname; der Kalser Bach fließt in seinem Mittelabschnitt in einem relativ ebenen Gebiet sehr ruhig dahin und sein Bett ist von Lachen und Pfützen begleitet, insbesondere im Frühjahr nach der Schneeschmelze und im Hochsommer nach ausgiebigen Regenfällen oder heftigen Gewittern. ${ }^{24}$ Von diesem (mittleren) Abschnitt könnte also die (slawische) Namengebung ausgegangen sein, wo sich auch andere Namen slawischer Herkunft finden, z. B. Lesach, Arnig und Staniska. Schon Karl Finsterwalder hat darauf hingewiesen, dass im unteren Bereich des Kalser Tales der historische Kern der slawischen Besiedlung zu suchen ist, ${ }^{25}$ liegen doch vier von den sechs Siedlungsnamen slawischer Herkunft in jenem Bereich (außerhalb liegt nur Ködnitz, Übersetzungsname zu romanisch Glor; der Name Kals selbst gilt heute für das ganze Tal und die Gemeinde). ${ }^{26}$

Eine zweite Deutungsmöglichkeit ergibt sich aus der Tatsache, dass die slawischen Wörter kalb und kaluža die gleiche Bedeutung haben können wie der in Tirol nicht seltene (ursprüngliche Flur-) Name Lizum (z. B. Axamer Lizum, Aussprache [litsúm], zu romanisch lozza 'Kot'), etwa 'Kotalm, Kotanger' ${ }^{27}$; mundartlich das Kot [khoat] bedeutet in Tirol überhaupt 'Lagerplatz des Viehs, wo viel Kot liegt ${ }^{28}$; der Boden der Viehleger ist bekanntlich überdüngt. In diesem Fall würde der Name Kals mit der Viehzucht zusammenhängen; gestützt wird diese Erklärung durch den Flurnamen Kaluse, eine ehemalige Viehweide im Bereich zwischen dem Burger und Ködnitztal. Eine romanische Herkunft des Namens Kals ist aus lautgeschichtlichen Gründen unwahrscheinlich und kann hier nicht näher ausgeführt werden.

Im Slawischen bedeuten die beiden in Frage kommenden Wortstämme kals und kaluža $a^{29}$ :

Schmutz, Unrat, Dreck;

Kot, Schlamm;

Kehrichtplatz

Viehtränke;

Lache, Pfütze;

Sumpf, Morast.

Davon abgeleitet sind kališće 'Lache, Pfütze' und kalina 'Schneeball (Pflanze): Viburnum, L.)'. Davon kommen für das Slowenische (und damit wohl auch für das

\footnotetext{
${ }^{24}$ Diese Ansicht wird durch die geomorphologischen und hydrologischen Gegebenheiten bei der Entstehung des Kalser Tales bestätigt (wie dies aus einem Vortrag von W. Wirkner, Limnologe aus Innsbruck, auf dem XII. Kalser Namenkundlichen Symposium hervorging; vgl. auch Wirkner 2002).

26 Finsterwalder 1990-1995: I 54.

26 Vgl. auch Odwarka - Pohl 1986-1998 und 2004.

27 Vgl. v. a. Finsterwalder 1990-1995: II 640.

28 Schatz 1955: 351.

29 Nach Šmilauer 1970: 87.
} 
Alpenslawische) nur die Bedeutungen 2, 3 und 5 in Frage sowie kališće. Ein Blick ins slowenische Wörterbuch ${ }^{30}$ weist folgende Wörter und Bedeutungen aus:

$k a \hat{t}$ 'Kot in Pfützen; flache Vertiefung, in welcher sich Regenwasser sammelt,

Lache; Viehtränke';

kalič 'kleine Lache';

kalíš, kalíšče 'Pfütze, Mistlache';

kalúža 'Kotlache, Pfütze'.

Ein Appellativ kalec ist nicht direkt greifbar, nur als Name, Kalec ${ }^{31}$, SN Kalce (pl., 4×), urkundl. 1499 Kalecz und $1356 \mathrm{Kalcz}^{32}$. Slaw. kaluža 'Kotlache, Pfütze' ist wahrscheinlich eine Ableitung von kalb. ${ }^{33}$

In Osttiroler Flurnamen weiters Gelischge (Alkus), n. [kə'liškə]; Ortsnamen mit diesen Elementen in Kärnten sind: Gall (Gem. St. Urban), Galling (1539 Gelicz, daher wohl zu kalič oder kališče), Kalischka (heute Ortsteil von Camporosso/Saifnitz, Kanaltal, Italien), in der Steiermark Kollisch (Gem. Stocking, Wildon) und in Salzburg der Hofname Galli (zweimal, Zederhaus und Sauerfeld). Beispiele aus Slowenien s. o. - Man kann also keinen ernsthaften Einwand gegen eine slawische Deutung von Kals vorbringen.

2.2. Von den insgesamt 13 Siedlungsnamen der Gemeinde Kals sind also immerhin 6 slawischer Herkunft, gegenüber je 3-4 deutscher bzw. romanischer Herkunft. ${ }^{34}$ Daraus kann man schließen, dass es vor dem Eintreffen der bairischen, deutsch sprechenden Siedler zu einer Symbiose des romanischen und slawischen Elements gekommen war, und zwar in der Weise, dass in das im Frühmittelalter ursprünglich rein romanische Gebiet die Alpenslawen eingewandert sind und sich dort niedergelassen haben, wo noch Platz war, ohne die bodenständige romanische Bevölkerung zu verdrängen. ${ }^{35}$ Dafür sprechen auch das Übersetzungspaar Ködnitz $\sim$ Glor und die zahlreichen slawischen Flurnamen gerade in exponierten Lagen. Erst im Laufe des 13. Jhdts. hat sich im Kalser Tal die deutsche Sprache durchgesetzt, doch gerade in den Namen spiegeln sich die alten Besiedlungsverhältnisse am besten wider. Die Siedlungsnamen slawischer Herkunft im Einzelnen:

Arnig (urkundlich 1288 Abernig, 1307 Awernichk): altslowenisch *avornik (< slawisch *avorbnikb), ursprünglich wohl Hofname, etwa 'Ahorner' zu slawisch (j)avorb, slowenisch javor 'Ahorn'.

Ködnitz: altslowenisch kotnica (< slawisch *kotbnica) 'Gegend im Winkel bzw. Winkelbach' zu slawisch *kotb 'Winkel', slowenisch kot; vgl. den bedeu-

\footnotetext{
${ }^{30}$ Pleteršnik s. v.

31 Bezlaj 1956: 246.

32 Auch Udolph (1979: 176) nennt *Kal-bcb und -bce.

33 So Udolph 1979: 186, andere Deutungsversuche bei Bezlaj 1982: 12.

34 Nach Pohl 2001: 6-8.

35 Vgl. Finsterwalder 1929: 241.
} 
tungsgleichen Nachbarort Glor (s. u.; urkundlich 1329 Anglar, 1428 Angular $<$ romanisch angulare 'im Winkel gelegen').

(Ober-, Unter-) Lesach (urkundlich 1244 Lescha, 1369 Lesach): altslowenisch *lešah (< slawisch *lěšaxъ), Lokativ zu *leš(an)e 'Waldbewohner', Einwohnername zu slawisch lěsz, slowenisch les 'Wald'.

(Ober-, Unter-) Peischlach (urkundlich 1329 Peuschler, 1428 Päuschlarn u. Peuschlärn): altslowenisch *pišljah (< slawisch *pyšl'axъ), Lokativ zu *pyšsl'(an)e, einem von slawisch *pyxati 'blasen, wehen' abgeleiteten Einwohnernamen, etwa 'Ort, wo der Wind weht', slowenisch pišlje; der urkundliche Beleg Peuschler zeigt den alten deutschen Einwohnernamen, die Form auf -arn/-ärn den Dativ, der syntaktisch dem slawischen Lokativ entspricht. Erinnert semantisch an deutsche Ortsnamen wie Windschnurn (Gemeinde Lendorf, Spittal an der Drau).

Staniska (mundartlich [niška], urkundlich 1545 zu Tanitsch, Tanitschga, 1692 Stanischga): altslowenisch *stanišće (< slaw. stan 'Standort, Lagerplatz' + Suffix -isko/-išće), alte Bezeichnung für Almhütten (slowenisch stanišče).

Und nun zu den Siedlungsnamen romanischer Herkunft:

Glor (amtlich auch Glor-Berg, urkundlich 1329 Anglar, 1428 Angular): aus romanisch angulare 'im Winkel gelegen'. - Auch das benachbarte Ködnitz (s. o.) hat die gleiche Bedeutung, beide Namen übersetzen einander.

Pradell $^{36}$ (Aussprache [pradél]): aus romanisch pratellu 'kleine Wiese'.

Elleparte (mundartlich [elepắrt(e)], älter [lepórtən]): aus romanisch (ad) illam partem 'jenseitiger Teil'. - Der Name

Lana kann entweder direkt auf romanisch labina 'Erdsturz, Lawine' bzw. *labinarium 'Lawinenzug' zurückgehen oder repräsentiert das aus letzterem entlehnte deutsch-mundartliche Lahner.

Die Siedlungsnamen deutscher Herkunft im Einzelnen:

Großdorf: der (heutige) Hofname Figer, urkundlich 1307 Zefig ( $<$ romanisch sub vico 'unter dem Dorf'), 1428 Fyger, weist darauf hin, dass Großdorf einst *Fig (< romanisch vicus 'Dorf') geheißen haben dürfte. Die Bezeichnung Großdorf (das wäre romanisch Vicone > Vigaun wie z. B. in Salzburg, es ist jedoch nur urkundlich villa maior 'größeres Dorf' bezeugt ${ }^{37}$ ) ist relativ jung, einst wird es wohl einfach Dorf geheißen haben, denn der ehemalige Gemeinbesitz von Kals-Großdorf wird mundartlich [dórfər ắlwe] (das ist Dorfer Alm, amtlich Dorfer Tal) genannt.

${ }_{36}^{36}$ So neuerdings amtlich geschrieben (statt früher Pradel).

371256 curiam apud Calts in villa maiori (vgl. Leimser 1998: 17). 
Burg und Unterburg (urkundlich um 1300 underbi̊rg, mundartlich [untər (dər) wurkh]): Hinweis auf ehemalige Burg ${ }^{38}$ sowie (ober Glor, s. o.) Berg.

Haslach: das ist die 'Haselstaudengegend', deutsch Hasel + bairisches Kollektivsuffix -ach.

2.3. Auch die Hofnamen der Gemeinde Kals enthalten Sprachgut aus allen drei Sprachen. ${ }^{39}$ Einige alte Hofnamen romanischer Herkunft sind u. a. Figer (s. o.), Golliseller (Glor-Berg, zu collicellu 'Hügelchen, Bichl'), Ranggetin(er) (Glor-Berg, von *runcu, - $a$ 'Rodung' + -ittu + -ina, vgl. ähnliche Namen wie Runchettin, Graubünden, und Rungett, Vorarlberg), Prädotzer (Großdorf, zu petra 'Stein' + -acea), Gliber (Großdorf, zu clivus 'Abhang, Hügel') sowie Labores (vormals Burg, zu luparias 'Wolfsgruben').

Nur drei Hofnamen sind sicher slawischer Herkunft, und zwar Perloger (Staniska, zu slowenisch prelog 'brachliegendes Ackerland, Anger, Lichtung'), Niederarnig(er) (Arnig, s. o.) und Oblasser (Peischlach, zu slowenisch $o b+$ plaz 'längs der Lawine, am Lahner'). Dazu kommt noch die romanisch-slawische Mischbildung Rantschnigg (abgekommen, Glor, romanisch *runca 'Rodung' + slowenisch -nik, ein Lagenamen bildendes Suffix wie deutsch -er im gleichbedeutenden Mischnamen Rantschner, Staniska).

Die überwiegende Mehrheit der Hofnamen ist jedoch deutscher Herkunft (über 80\%). - Von den insgesamt über 1300 Flur-, Berg-, Gewässer-, Hof- und Siedlungsnamen im Kalser Tal kann man heute etwa 65\% bairisch, 27\% ladinisch, 6\% slowenisch und 2\% vorrömisch deuten. Das Kalser Tal ist wohl die einzige Gegend Österreichs, wo alle drei Hauptsprachen unserer Zeitrechnung (Ladinisch / Romanisch, Altslowenisch / Slawisch und Bairisch / Germanisch) in gleicher Weise in der Namengebung konstitutiv waren.

3. Ein anderes Bild bietet die benachbarte Gössnitz, amtlich Gößnitztal im oberen Mölltal (Gem. Heiligenblut). ${ }^{40}$ Hier haben wir ein primär slawisch benanntes Gebiet vor uns, das zunächst von Ziegenhirten (genauer: Bauern, die Ziegen gehalten haben) in Besitz genommen wurde. Der Gewässername Gößnitz, nach ihm ist auch das Tal so benannt, ist als 'Ziegenbach' zu deuten, etwa slow. *koznica (zu koza

38 Oder Fluchtburg. Vgl. als Parallele zu Burg den von Finsterwalder 1990-1995: I 54 als 'Burgstall' gedeuteten Flurnamen Geschgelier [keškelî́r] (zu romanisch castellu 'Kastell, Burg'). Dieser ist aber aus lautlichen Gründen eher auf romanisch *costellaria (zu costa 'Seite, Rippe', in der Toponymie 'Hangrippe, länglicher Bergvorsprung; Berglehne', mehreren Namen in Kals zugrundeliegend, z. B. Göscht(bachl), Göschteloi) zurückzuführen. - Es ist wegen der Aussprache [wurkh] auch slawisch vrbxb ( $>$ slowenisch $v r h$ ) 'Gipfel, Anhöhe' vermutet worden.

40 Auf Grund des Projektes „Die unsichtbare Geschichte der Landschaft - Flurnamen und Toponymie im Gössnitztal“. Pilotstudie im Auftrag der Nationalparkverwaltung Hohe Tauern Kärnten, Projektidee und Konzeption Kirsten Melcher, Projektleitung Heinz Dieter Pohl, Mitarbeiter H. Guggenberger, H. Hoffert, B. Menne, R. Unterguggenberger. 
'Ziege'), in der österreichischen Toponymie sehr geläufig; eine Gößnitz kommt in Österreich dreimal vor. Dazu passen die Flurnamen Kasaze / Kasarn: da die Beschreibung dieser beiden (benachbarten bzw. ineinander gehenden) Fluren keinen Anhaltspunkt auf das Vorhandensein von Almhütten (ma. Kaser, von rom. casa) bietet, muss eine andere Deutung gesucht werden. Daher liegt - wie beim Talnamen selbst - slow. koza 'Ziege' nahe. Wahrscheinlich handelt es sich um ehemalige Ziegenweiden, slow. kozarica (von kozar 'Ziegenhirt'), kozara 'Ziegen-, Schafhürde' usw. Allerdings kommt koza in der Bergnamengebung auch in übertragener Bedeutung vor, z. B. slow. Kozjak 'Geißberg', kozji hrbet 'Geißrücken' (ähnlich wie dt. Geiß in Geißberg oder Bock z. B. in Bosruck). Letzteren Flurnamen (ma. Goaßrucken) gibt es in dt. Namensform auch in unserem Untersuchungsgebiet. Auch Ziegen werden gemolken; an den Mittelpunkt dieser Wirtschaftsform erinnert Malesischk. Diese wird als Gebiet mit Almhütten (Kasern) beschrieben, daher kann man davon ausgehen, dass hier immer schon das Vieh gemolken wurde. Somit liegt diesem (auch auf den ersten Blick slaw. anmutenden) Namen ein slow. *molzišče 'Ort, wo man melkt; Melkerei, Melkstätte' zugrunde. Das slow. (Örtlichkeiten bezeichnende) Wortbildungselement -išče < slaw. *-išće lautet bei früher Übernahme ins Dt. bzw. allgemein in Osttirol und Oberkärnten meist -ischk (oder geschrieben auch -isk). - Weitere Namen:

Albitzen: Da das ma. Wort (westbair.) ålbe bzw. (gemeinbair.) ålm nie mit dem slaw. Suffix -ica kombiniert wird, bietet sich als Ausgangsform ein slow. jalovica an, zu jalov 'galt (= keine Milch gebend, also Bezeichnung für das Jungvieh), (auch) unfruchtbar'. Hier dürfte es sich also um einen aufgewanderten Flurnamen handeln, der einst eine 'Galtalm' bezeichnete. Da slow. jalovica auch 'unfruchtbarer Erdboden' bedeuten kann, ist eine Primärbenennung ebenfalls möglich. Fehlen des prothetischen $j$ - in Namen slow. Ursprungs ist - wie bereits erwähnt - in Oberkärnten und Osttirol die Regel.

Aureon: Dieser Name könnte auf ehemalige Ahornbestände hinweisen. Das slow. Wort (j)avor- 'Ahorn' ist in der österreichischen Namengebung sehr verbreitet, man denke an den Ortsteil von Kals am Großglockner Arnig, an den Bergnamen Auernig bei Mallnitz sowie den ähnlich klingenden Namen Auere in der Innerfragant usw. Als Ausgangsform ist ein slow. (j)avorovina o. ä. anzusetzen. Die slow. Grundform dürfte im Flurnamen Aureboden vorliegen.

Zopenitzen: Dieser Name beruht auf einem slow. sopotnica (zu sopot 'Wasserfall, -dunst'), also 'Wasserfallbach' (wie slow. Sopotnica / dt. Sapotnitza, Loibltal). 
Retschitz: Dieser Name beruht auf slow. rečica 'kleiner Bach' (zu slow. reka 'Bach, Fluss', Namensparallele slow. Ročica / dt. Rotschitzen, Köttmannsdorf u. Viktring). ${ }^{41}$

4. Einige interessante Bergnamen aus dem Nationalpark Hohe Tauern: ${ }^{42}$ Auernig [K 44], aus frühslawisch *avorbnikb 'Ahornberg' (wie Jauerling in Niederösterreich).

Daberkögele [T 39]: Daber- zu slawisch *dbbrb 'Schlucht', slowenisch deber, daber 'Talschlucht', davon das Osttiroler Mundartwort daber (in Kals [dāwa, dāwər]) 'Klamm' bzw. tâwer 'Stellen, an denen ein Bach entspringt' (Defereggen) bzw. 'mit Gesträuch bewachsener Fleck, der an den Bach steil angrenzt; abhängiges Wiesenstück, Rain an einem Bach' (Virgen).

Dabernitzkogel [T 36]: zum slawischen Flurnamen *dbbrbnica, von *dbbrb, vgl. Daber-wie vor.

Debantgrat [T 41]: zum Debanttal hin gelegen (der Name Debant beruht auf slawisch *děvina 'Jungfrauenort', urkundlich 1274 Dewin, auf ins Christentum übernommene alte Frauenkulte hindeutend).

Deferegger Riegel [T 36]: nach dem Defereggen(tal) (Gem. St. Jakob i. D., St. Veit i. D., Hopfgarten), dieses urkundlich 1155-64 Toberegge, 1213 Douerich, < frühslowenisch *Dobrik'e, von einem mit slawisch *Dobr- 'gut' beginnenden Personennamen abgeleiteter Gegend- oder Siedlungsname. Die Schreibung Defereggen(tal) ist die amtliche, die mundartliche Form lautet Defreggen.

Gamolnigspitz(e) [K 44]: von einem Flurnamen slowenisch *gomilnik zu gomila 'Erdhaufen, Grabhügel'.

Gesselköpfe (Vorderer Gesselkopf) [K/S 42]: zur Gesselwand (< Gössel- < slowenisch kozlji oder kozlov zu kozel 'Ziegenbock', auch 'Gamsbock'), die in einem Gebiet liegt, wo sich gerne Gämsen aufhalten.

Glödis(spitze) [T 41]: < frühslowenisch *glodišće 'Ort, der vom Wasser zernagt ist' (zu slowenisch glodati 'nagen'), doch lautlich schwierig. Lautlich wahrscheinlicher und wortbildungmäßig möglich ist der Ansatz *glodež mit ähn-

${ }^{41}$ Neben dem slawischen Element nimmt sich das romanische bzw. vorrömische Substrat sehr bescheiden aus, u. a. Fran, die als Lawinenstrich beschrieben wird, es ist zunächst an rom. *fra(gi)na 'Erdrutsch, Bergsturz' zu denken. Die Beschreibung als Geländevorsprung lässt auch eine Namendeutung wie Frohntal zu, aus rom. voragines (pl. zu lat. Vorago 'Schlund, Abgrund'). Die Ausgangsform muss in beiden Fällen *frän(e) gewesen sein. Weiters das Appellativ Balfen, Palfen (Balm, Palm) 'Felsen; Felszacken, -stufe, -vorsprung, -höhle; überhängender Fels', das über das Rom. ins Dt. gelangt, Substratwort (vorröm. *péllawo- 'Fels'). Eher typisch für Tirol, doch bis nach Oberkärnten reichend, z. B. Hochpalfennock im Nockgebiet. In Kals (Osttirol) als ,,deutsches“ Appellativ, z. B. Balfen (Ortsteil Lesach) oder Weißer Balfen (Ortsteil Dorfer Tal), „deutsches“ Pendant zum benachbarten Bergnamen Gradalfe (< rom. croda alba 'weißer Felsen').

42 Weitere Literatur in meinen Arbeiten Pohl 1996, 1999, 2001, 2005a-b u. Odwarka -Pohl 2004. Im Internet unter der URL http://members.chello.at/heinz.pohl/Bergnamen.htm. 
licher Bedeutung (allerdings bezeichnet das Wort glodež auch mythologische Wesen).

Gollspitz [T 39]: enthält entweder romanisch collis 'Berg, Hügel' oder ist ein 'Kahlenberg' (von slowenisch gol 'kahl').

Golz [T 40]: die Golz < slawisch golica 'kahle Stelle', Bezeichnung für glatte, wiesenbedeckte Fluren und Berge).

Gößnitzkopf [K/T 41]: nach dem Gewässernamen Gößnitz, dem auch das Gößnitztal seinen Namen verdankt und der als 'Ziegenbach' zu deuten ist, etwa slowenisch *koznica (zu koza 'Ziege'), in der österreichischen Toponymie sehr geläufig; eine Gößnitz kommt in Österreich dreimal vor.

Gramúl, Gremúl [T 40]: zu slowenisch krmol, krmulja 'Felsvorsprung, Anhöhe' oder grmulja 'Haufen, Klumpen' (nach Bezlaj 1976: 179 eher gemeinslawisch, dies aber könnte auch eine romanische Entlehnung sein, vgl. den ebenfalls in Osttirol liegenden Bergnamen Gumriaul, zu gall. comboros 'Verhau, Geflecht', französisch combres 'Schutt', mhd. kumber 'ds.').

Ködnitztal [T 40]: nach dem Ködnitzbach < slawisch *kotbnica 'Gegend im Winkel bzw. Winkelbach' zu *kotb 'Winkel' > slow. kot; vgl. den bedeutungsgleichen Nachbarort Glor, urkundlich 1329 Anglar, 1428 Angular < romanisch angulare 'im Winkel gelegen'. Dazu auch der Name des Ortsteils Ködnitz der Gem. Kals a.Gr.

Lanischeck, -kees [K 44]: zu slowenisch lanež 'Bergrücken, Kamm, Grat (schneereich und/ oder schwer zu übersteigen)'. Herkunft dieses Wortes wohl von slowenisch lani 'voriges Jahr', vgl. das Paar deutsch Ferner $\sim$ romanisch vedretta, beide 'Altschnee', von lateinisch vetus 'alt'. Das deutsche Ferner hängt mit Firn 'vorjähriger Schnee', mittelhochdeutsch virne, vern(et) 'im vorigen Jahr' zusammen, vgl. auch bairisch ferten 'ds.'. Es könnte also eine südalpine romanisch-deutsch-slowenisch semantische Gleichung vorliegen wie dies u. a. auch bei „Dachboden“ (südbairisch Unterdåch, slowenisch podstrešje, furlanisch sotèt $\sim$ romanisch subtum tectum 'unter dem Dach') und 'Frühling' (südbairisch Auswart, slowenisch mundartlich vigred, furlanisch inšude romanisch (*in-) exitus 'Ausgang') der Fall ist. Somit wäre die Grundbedeutung von slowenisch lanež 'mit Firn überzogener Bergrücken oder Kamm, Gletscher', die sich mit dem Rückgang der Vergletscherung zu 'schwer zu überwindender Bergrücken usw.' gewandelt hat. Dieses Appellativ kommt auch zwei Mal in Slowenien in den Kamniške in Savinjske Alpe / Steiner und Sanntaler Alpen vor, wo man sich vorstellen kann, dass bei kälterem Klima der Firn lange liegen blieb. Dazu auch der Bergname Wainasch / Vajnež in den Karawanken.

Lasörling [T 36]: urkundlich 1670 Lasernik, demnach ein umgeformtes slowenisches lazarnik zu lazar 'Reuter, Rodender' als Flurname oder Hofname.

Lasörlinggruppe [T 36]: s. Lasörling; dieser südöstlich der Lasnitzen(alm) liegende Berg hat der ganzen Gebirgsgruppe den Namen gegeben. 
Leibnitztörl (Westliches Leibnitztörl) [T 41]:

Mirnitzspitze (Große, Kleine Mirnitzspitze) [T 41]: vom Gewässernamen Mirnitzbach < slawisch *nyrbnica von *nyrati (se) '(unter)tauchen, unter die Erde schwinden' bzw. *nyrěti 'hervorströmen, einsickern'; *nyrbn- im Slowenischen ziemlich regelmäßig > mirn- umgeformt, vgl. Namen wie Mirna, Mirnik in Slowenien.

Muntanitz (Kleiner und Großer Muntanitz) [T 39]: wohl nach dem Muntanitzbach, möglich ist sowohl ein lautlich sehr altertümliches altslowenisches *motbnica 'Trübenbach' (vgl. slowenisch Motnica > deutsch Metnitz) als auch ein slawisiertes romanisches montan- 'Berg-' + slawisch -ica (denkbar, doch wenig wahrscheinlich, ist auch ein rein romanisches *montanitia, -ities etwa 'Bergland, Gebirge').

Nussingkogel [T 39]: früher Mussi(n)g, entweder zu romanisch *mužina 'Steinhaufen' oder zu slawisch muža 'Sumpf, Morast' (in letzterem Falle ein „aufgewanderter" Flurname einer nassen Wiese).

Pasterze [T 40]: dieser Name aus dem Sagengut her erklärbar, analog wie die Übergossene Alm in Salzburg. Auszugehen ist von slowenisch pastir 'Hirt' (auch in mundartlich Pasteier 'kleine Almhütte', Liesertal, enthalten), etwa *pastirica 'Hirtengegend', verbunden mit der Vorstellung, dass unter dem Gletscher einst ein üppiges Almgebiet lag, was im unteren Bereich früher durchaus möglich war (vgl. 1554 wise ... in der Pasterze). Das untere Ende der Pasterze ist die Margaritze, mundartlich Magarétzen, die 'magere Etze', heute ein karges Gebiet.

Perschitzkopf (Hoher, Östlicher Perschitzkopf) [K/T 41]: nach dem Perschitzbach (Gem. Nussdorf-Debant), urkundlich. 1583 Perschitzpach, zu slawisch *pъršica etwa 'Spritzbach' (vgl. slowenisch pršica 'Nieselregen').

Perschitzschneid [K/T 41]: wie vor.

Petzeck [K 41]: die Erklärung des Namens dieses Berges lässt zwei Deutungen zu, entweder man betrachtet Petz- als slawisch (vgl. slowenisch peč 'Ofen, Felsen') wie die Petzen / Peca (s. Petzen) und den Petsch (s. Ofen) in den Karawanken oder man betrachtet ihn als romanischen Namen, vgl. die vielen Bergnamen in Westösterreich, Südtirol und der Schweiz mit Piz. Dieses kommt von rätoromanisch bzw. ladinisch piz 'Spitze' (aus romanisch pittsunklarer Herkunft). Obwohl in der Schobergruppe slawische Namen recht häufig sind, denke ich doch eher an die romanische Lösung (es handelt sich ja um einen markanten Berg, erweitert um deutsch $E c k$ 'hervorspringende Erhebung').

Schleinitz [T 41]: ist möglicherweise ein aufgewanderter Flurname, slowenisch slivnica 'Pflaumen-, Zwetschkengegend', auch Gewässername. Denkbar ist aber auch ein Zusammenhang mit slowenisch skljuvati 'mit dem Schnabel zerhacken' als Flurname *skljuvnica (etwa) 'zerhackte Gegend' (wegen der 
zerrissenen Felsen des Berges, ein vergleichbarer Namen deutscher Herkunft ist das Ghackte im Hochschwabgebiet).

Sunzkopf [T 39]: so von Matrei i. O. aus genannt, in Kals Kendl(spitz(e), -kopf), wohl < slawisch *zobbcb 'kleiner Zahn; Zahnkopf' zu slowenisch zob 'Zahn', in der Toponymie 'stark zugespitzte Steinform; alleinstehender schlanker, hoher Felsen (auch gerippt)'.

Wangenitzen bzw. Wangenitztal [K 41]: geht auf frühslawisch *vagelbnica (über *vegelbnica > slowenisch vogelnica) 'der aus dem Winkel kommende Bach', semantisch mit dem Ködnitztal übereinstimmend.

Woisgenkopf [K/S 44] (auch mit -sk- geschrieben), zu slawisch vysokъ 'hoch'.

Wurtenkees [K 42]: enthält wohl das slowenische Adjektiv vrten 'Garten-, zum Garten gehörig' zu vrt 'Garten, eingefriedete Bergwiese, Mahd', wohl aufgewanderter Flurname.

Zettalunitzkees [T 36]: kein urkundlicher Beleg, < slawisch *sedъlovbnica 'Sattelkees, der vom Sattel (slowenisch sedlo) herunterkommende Gletscher'.

Zopenitzen [K 41]: dieser Name beruht auf einem slowenischen sopotnica (zu sopot 'Wasserfall, -dunst'), also 'Wasserfallbach' (wie folg. und slowenisch Sopotnica / deutsch Sapotnitza, Loibltal).

Zopetspitze [T 36]: nach den Wasserfällen des Timmelbaches, vgl. Zopatnitzenalm und -bach (urkundlich 1500 Zopotnitzen, < slawisch *sopotbnica 'Wasserfallbach' zu sopotb 'Wasserfall' (> slowenisch sopot); dieser vom Bergersee herabstürzende Bach weist mehrere Wasserfälle auf.

Zunig (Großer, Kleiner Zunig) [T 36]: wie Zunigalm, -bach, -see zum frühslowenischen Flurnamen *zunikb zu slowenisch zuni bzw. zunaj 'draußen', also etwa 'außerhalb, abseits gelegen'.

\section{Literatur}

Bezlaj 1956: $\quad$ F. Bezlaj, Slovenska vodna imena I, Ljubljana

Bezlaj 1976, 1982, 1995: F. Bezlaj, Etimološki slovar slovenskega jezika I-III, Ljublana

Finsterwalder 1929: K. Finsterwalder, Ueber Tauernnamen, Zeitschrift für Ortsnamenforschung 5, 228-242

Finsterwalder 1990-1995: K. Finsterwalder, Tiroler Ortsnamenkunde I-III, Innsbruck

Holzer 1997: $\quad$ G. Holzer, Die Herkunft des $(t) z$ in Kollmitz etc. (*kalamantiā), Kobenz $(*$ kumbantiā) und Lafnitz (*albantiā), Österreichische Namenforschung 25 (= Festschrift Odwarka), 81-103

Holzer 2001: $\quad$ G. Holzer, Die Slaven im Erlaftal. Eine Namenlandschaft in Niederösterreich, Wien

Holzer 2002a: G. Holzer, Zur Sprache des mittelalterlichen Slaventums in Österreich. Slavisch unter bairischem Einfluss, Wiener Slavistisches Jahrbuch $48,53-73$

Holzer 2002b: G. Holzer, O leksiku slavenskoga supstrata u Austriji. II. Pregled o građi, Folia onomastica Croatica 11, 95-102

Holzer 2002c: $\quad$ G. Holzer, Landschaft und Siedlung im slavischen Frühmittelalter, in: P. Anreiter - P. Ernst - I. Hausner, Hg., Namen, Sprachen und Kultu- 
ren / Imena, jeziki in kulture, Festschrift für Heinz Dieter Pohl zum 60. Geburtstag, Wien, 387-397

Hornung 1964:

M. Hornung, Mundartkunde Osttirols, Wien

Kronsteiner 1975:

O. Kronsteiner, Die alpenslawischen Personennamen, Wien

Leimser 1996:

Leimser 1998:

H. A. Leimser, Geschichte von Kals (Diplomarbeit), Innsbruck

H. A. Leimser, Geschichte von Kals am Großglockner durch die Jahrhunderte, Kals am Großglockner (Gedruckte Fassung von vorigem mit Anhang)

Mader 1986:

B. Mader, Die Alpenslawen in der Steiermark, Wien

Neweklowsky 1997: G. Neweklowsky, 224. Deutsch-Kroatisch. Kontaktlinguistik. Ein internationales Handbuch zeitgenössischer Forschung, 2. Halbband, Berlin - New York, 1821-1827

Odwarka - Pohl 1986-1998: K. Odwarka - H. D. Pohl, Materialien zu einem Namenbuch von Kals (Osttirol), 5. Teil: Hofnamen, Österreichische Namenforschung 14-26 (Aufsatzreihe in 5 Teilen, Zusammenfassung: Odwarka - Pohl 2004)

Odwarka - Pohl 2004: K. Odwarka - H. D. Pohl, Alle Kalser Namen auf einen Blick: Register $\mathrm{zu}$ allen bearbeiteten und erhobenen Namen des Kalser Tales mit kurzer Erläuterung (Herkunft), in: H. D. Pohl, Hg., Kalser Namenbuch, Österreichische Namenforschung Sonderband, Wien, 11-52

Pleteršnik 1894: $\quad$ M. Pleteršnik, Slovensko-nemški slovar I-II, Ljubljana (Nachdruck 1974, Neudruck 2006)

Pohl 1996:

H. D. Pohl, Die Osttiroler Ortsnamen slawischer Herkunft, Österreichische Namenforschung 24, 39-64

Pohl 1996a: $\quad$ H. D. Pohl, Zur Sprache der Freisinger Denkmäler. Beziehungen zwischen der Sprache der Freisinger Denkmäler und den Ortsnamen Oberkärntens und Osttirols alpenslawischer bzw. slowenischer Herkunft, in: Zbornik Brižinski spomeniki, Ljubljana, 311-321

Pohl 1996b:

H. D. Pohl, Die Osttiroler Ortsnamen slawischer Herkunft, Österreichische Namenforschung 24, 39-64

Pohl 1997:

H. D. Pohl, 222. Österreich. 223. Deutsch-Slowenisch. Kontaktlinguistik. Ein internationales Handbuch zeitgenössischer Forschung, 2. Halbband, Berlin - New York, 1797-1812

Pohl 1999: H. D. Pohl, Slawische (slowenische) Bergappellativa in der österreichischen Oronymik. Studia celtica et indogermanica, in: Festschrift für Wolfgang Meid, hg. v. P. Anreiter - E. Jerem, Budapest, 331-342

Pohl 2000: $\quad$ H. D. Pohl, Kärnten - deutsche und slowenische Namen. Koroška slovenska in nemška imena. Kommentiertes zweisprachiges Verzeichnis der Siedlungs-, Berg- und Gewässernamen, Österreichische Namenforschung 28, Heft 2-3; Lizenzausgabe in der Reihe Studia Carinthiaca Bd. XIX, Klagenfurt

Pohl 2001: $\quad$ H. D. Pohl, Kals am Großglockner. Ein kleiner namenkundlicher Führer, Kals am Großglockner

Pohl 2002a: $\quad$ H. D. Pohl, Siedlungsgeschichte und Überlieferung von Ortsnamen slowenischer Herkunft in Osttirol und Kärnten (mit Ausblicken aufs übrige Österreich). Ortsnamen und Siedlungsgeschichte (Akten des Symposiums in Wien vom 28.-30. September 2000, hg. v. P. Ernst - I. Hausner - E. Schuster - P. Wiesinger), Heidelberg, 177-189

Pohl 2002b: $\quad$ H. D. Pohl, Aus Kärntens sprachlicher Vielfalt. Beiträge zur Kärntner Mundart- und Namenkunde, Fidibus, Zeitschrift für Literatur und Literaturwissenschaft 29

Pohl 2004a: $\quad$ H. D. Pohl, Die Sprache der Kärntner Küche / Jezik koroške kuhinje. Ein Lexikon mit Ausblicken auf die österreichische und internationale Küche (für alle, die gerne kochen und wissen wollen, woher viele Küchenausdrücke kommen und was sie bedeuten), Klagenfurt

Pohl 2004b: $\quad$ H. D. Pohl, Hg., Kalser Namenbuch, Österreichische Namenforschung (Sonderband) Wien 
Pohl 2005a:

H. D. Pohl, Kärntner zweisprachige Ortsnamen als verbindendes Kulturgut. Filled with many-splendored words. Papers on Culture, Language and Literature in Honour of Prof. Dr. Fritz Hans König, edited by Alicja Witalisz, Dieter Jandl, Karl Odwarka, Heinz Dieter Pohl and Władysław Witalisz, Krosno (PL), 281-288

Pohl 2005b:

H. D. Pohl, Die Slavia submersa in Österreich: ein Überblick und Versuch einer Neubewertung, Linguistica XLV - Ioanni Orešnik septuagenario in honorem oblata I, Ljubljana, 129-150

Ramovš 1936:

F. Ramovš, Kratka zgodovina slovenskega jezika I, Ljubljana (Nachdruck 1995)

Schatz 1955:

J. Schatz, Wörterbuch der Tiroler Mundarten, Innsbruck ( $\left.{ }^{2} 1993\right)$

Šmilauer 1970:

V. Šmilauer, Př́ručka slovanské toponomastiky - Handbuch der slawischen Toponomastik, Praha

Snoj 2003:

Udolph 1979:

WBÖ:

Wirkner 2002:

M. Snoj, Slovenski etimološki slovar, druga, pregledana in dopolnjena izdaja, Ljubljana [1997]

J. Udolph, Studien zu slavischen Gewässernamen und Gewässerbezeichnungen, Heidelberg

Wörterbuch der bairischen Mundarten in Österreich, Wien 1963 ff.

W. Wirkner, Die Besiedlung des Kalser Tales im Lichte hydrologischer Gegebenheiten, in: P. Anreiter - P. Ernst - I. Hausner, Hg., Namen, Sprachen und Kulturen / Imena, jeziki in kulture. Festschrift für Heinz Dieter Pohl zum 60. Geburtstag, Wien, 855-863

Zdovc 1972: $\quad$ P. Zdovc, Die Mundart des südöstlichen Jauntales in Kärnten, Wien

Abkürzungen (in Auswahl)

(außer allgemein bekannte Abkürzungen und Sprachbezeichnungen, bei denen nur die Silbe ...isch fehlt)

$\begin{array}{ll}\text { A } & \text { Akkusativ } \\ \text { Bez. } & \text { Bezirk } \\ \text { BN } & \text { Bergname } \\ \text { D } & \text { Dativ } \\ \text { dt., Dt. } & \text { deutsch, Deutsch } \\ \text { FN } & \text { Flurname } \\ \text { G } & \text { Genitiv } \\ \text { GB } & \text { Gerichtsbezirk } \\ \text { Gem. } & \text { Gemeinde } \\ \text { GN } & \text { Gewässername } \\ \text { HN } & \text { Hof- bzw. Hausname } \\ \text { I } & \text { Instrumental } \\ \text { idg. } & \text { indogermanisch } \\ \text { K } & \text { Kärnten } \\ \text { L } & \text { Lokativ } \\ \text { Lpl. } & \text { Lokativ Plural } \\ \text { lat. } & \text { lateinisch } \\ \text { ma. } & \text { mundartlich } \\ \text { mhd. } & \text { mittelhochdeutsch } \\ \text { NÖ } & \text { Niederösterreich } \\ \text { ON } & \text { Ortsname } \\ \text { ÖNf } & \text { Österreichische Namenforschung (Zeitschrift) } \\ \text { pl. } & \text { Plural } \\ \text { PN } & \text { Personenname } \\ \text { rom. } & \text { romanisch } \\ \text { S } & \text { Salzburg } \\ \text { sg. } & \text { Singular } \\ \end{array}$


skr.

slow.

SN

St

$\mathrm{T}$

urk. serbisch und kroatisch (einschließlich bosnisch)

slowenisch

Siedlungsname

Steiermark

Tirol

urkundlich

Die in eckige Klammern gesetzten Nummern bedeuten folgende Gebirge:

36. Venedigergruppe

39. Granatspitzgruppe

40. Glocknergruppe

41. Schobergruppe

42. Goldberggruppe

43. Kreuzeckgruppe

44. Ankogelgruppe

\section{Heinz Dieter Pohl}

Universität Klagenfurt

Institut für Sprachwissenschaft und

Computerlinguistik

Universitätsstraße 65-67

A-9020 Klagenfurt/Celovec

Heinz.Pohl@uni-klu.ac.at

heinz-pohl@chello.at 
